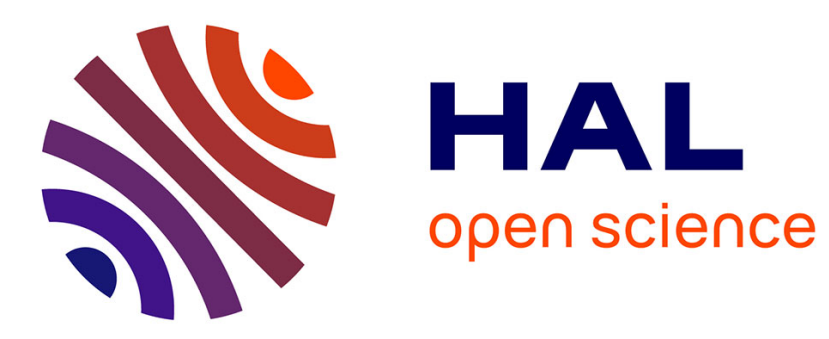

\title{
When overconfident agents slow down collective learning
}

\author{
Juliette Rouchier, Emily Tanimura
}

\section{To cite this version:}

Juliette Rouchier, Emily Tanimura. When overconfident agents slow down collective learning. SIMULATION: Transactions of The Society for Modeling and Simulation International, 2012, 88 (1), pp.33-49. 10.1177/0037549711428948 . hal-00623966

\section{HAL Id: hal-00623966 \\ https://hal-paris1.archives-ouvertes.fr/hal-00623966}

Submitted on 15 Sep 2011

HAL is a multi-disciplinary open access archive for the deposit and dissemination of scientific research documents, whether they are published or not. The documents may come from teaching and research institutions in France or abroad, or from public or private research centers.
L'archive ouverte pluridisciplinaire $\mathbf{H A L}$, est destinée au dépôt et à la diffusion de documents scientifiques de niveau recherche, publiés ou non, émanant des établissements d'enseignement et de recherche français ou étrangers, des laboratoires publics ou privés. 


\title{
When overconfident agents slow down collective learning
}

July 6, 2011

\author{
Juliette Rouchier et Emily Tanimura \\ GREQAM - CNRS \\ 2 rue de la Charité, 13002 Marseille, France \\ juliette.rouchier@univmed.fr emily.tanimura@gmail.com
}

\begin{abstract}
This paper presents a model of influence where agents' beliefs are based on an objective reality, such as the properties of an environment. The perception of the objective reality is not direct: all agents know is that the more correct a belief, the more successful the actions that are deduced from this belief. A pair of agents can influence eachother when they perform joint action. They are not only defined by individual beliefs, but also idyosynchratic confidence about their belief - this means that they are not all willing to engage in an action with agents with a different belief and to be influenced by them. After exploring the dynamics of our influence and learning system with agents that have the same confidence, we study heterogenous confidence among agents. We show here that the distribution of confidence in the group has a huge impact on the speed and quality of collective learning and in particular that a small number of overconfident agents can prevent the whole group from learning properly. keywords: belief dissemination, bounded-confidence, social influence, simulation agents, Agent-Based Computational Economics
\end{abstract}

\section{Motivation}

\subsection{A learning model with influence}

In this paper we present results of an influence model within a group of agents in a situation that has not, to the best of our knowledge, been studied before: when the belief about which agents influence eachother is based on an objective reality. As a first approximation, we chose to represent the objective reality as an environment with unchanging characteristics. As we will illustrate, this modelling is well adapted to represent some elements of the actual environment of humans. This addition implied that we had to choose a representation of how agents can access the information about the properties that constitute their belief, and we chose for this an indirect 
representation of "learning through action" - when acting successfully, an agent tends to believe that he should not change its belief; when failing, it questions its belief which might not be that accurate. The constitution of an influence model implies that agents interact by pair and influence eachother with a potentially heterogenous strength. The process of learning is such that the action is performed by a pair while only one belief is used (and hence tested). The success of an action makes the belief that was used reinforced for the pair. The agents are heterogenous in their acceptance to act following someone else's belief, so we integrate the usual notion of bounded confidence. We then consider that an interesting point is to study idyosynchratic confidence for individuals, and show that overconfident agents do not only have difficulties learning themselves but that they can also deprive others of opportunities to learn.

As a starting point, we consider an environment upon which agents do not have any impact at the local scale through their action and which is unchanged in the time-scale of the simulation. For example, one could consider that the environment is climate, and the representation we give of the environment is an equation about the evolution of its state, considering current state. The action agents have to choose could depend on next day climate, and they use one prediction for it - if they succeed, they think that the equation they used is right and if they fail, they think that the equation is wrong. Another idea could be farmers who want to share water and have to dig a well. For this they use their knowledge about where water is situated in the underground. The reinforcement learning would be the same as the previous one. The belief that agents have of the environment can be described as parameters that fit specific representation, based on a common framework (shape of an equation, or standard map upon which the relevant information are situated).

We choose an environment which properties cannot be directly accessed by agents, who have to assess their belief through observed results of action because this process corresponds to what humans actually do: acting to test our believes is our way to acquire knowledge (MV72).

Our learning is based on an joint action: agents collaborate in actions and influence eachother. It is linked to the idea that most acquisition of knowledge takes place through interaction (Mos79). This slighlty complex model, which will be described later, is such that we can integrate at the same time the experimental aspect of learning and interactive aspect through social influence. The idea that agents's confidence has an impact on their learning is linked to the notion of bounded-confidence which is common in social influence literature and finds ground in psychological observations as well, where a large field explores the influence of overconfidence on learning and performance. In our model, since we had to integrate the "action phase" in the learning about the objective reality, we have to re-write the usual algorithm for boundedconfidence, as is described in section 2 .

The main result of our study is that the presence of some very confident agents, who cannot be influenced by others, slows down the learning of the society as a whole and can even stop it. One can identify thresholds in the number of overconfident agents, 
that produce different patterns in the simulations. In particular, results depend on the probability of meeting among agents that can influence each other. For example we spotted that about $20 \%$ of the population of agents that are sensibly more confident than others is the worst configuration in our model.

The following subsections are meant to justify some of our modelling choices. Then, in following sections we present the simulation model, describe the dynamics of the simulation that imply our main result and then make some variation in the parameters to show the stability and limits of the learning and influence model. We eventually show how a few over-confident agents can disturb collective learning. This result suggests an interesting interpretation regarding the need for an open-minded population in a context of complex learning.

\subsection{Beliefs and choice of action}

In this paper we separate beliefs and preferences as an element of choice for action. This relies on the most basic model in orthodox thinking in game-theory or decision theory ((Kre90)). An action (sometimes called optimal strategy) is chosen according to aims (preferences) and beliefs. An agent that wants to reach an aim has images about how this aim could be reached, which pushes him to choose an action to reach it. It is to be noted that with this simple model of decision, two agents who have opposite preferences and different beliefs can choose the same action to attain their aim. Following the same logic, two agents with the same aim but different belief can choose a different actions to attain their aim.

In our setting, we only work on believes and their assessment. In this respect, we consider that all agents agree on the aim but differ in beliefs, and hence in the choice of the correct action to perform. This is where a comparison of confidence in one's belief has a role to play when a pair of agents decides on an action.

We are in particular interested in drastically separating learning and preferences in the model because we want to eliminate strategic aspects agents and differences in the perception of success or failure of the action. Here, agents may think that their beliefs are wrong if their aim is not achieved, and that it is right if their aim is achieved. The representation of learning corresponds to agents that conceive their actions as experiments, but who do not accumulate an individual memory of their past beliefs and the successes of their past actions. In that sense we position closer to "social influence" literature (agents instantly forget their previous belief at each step) than learning literature (where the relevance of different belief can be compared over time).

\subsection{Influence}

Social influence has been studied quite extensively by simulation and can be based on three usual representations.

In a very popular approach, the one that is developed by phycisists, agents' opinion can take only two values: 0 or 1 . This enables society to be conceived as a set of 
positively and negatively charged molecules in a network, that influence eachother. Ising fields are the calculating device of the evolution of the agents ((Gal97)). What can be proven formally in this type of system is, for example, the great influence of the distribution of initial opinions (SWS00), the topology of the network (CR05) (Sta05), as well as an exogenous informational shock in the system (FS06). One can discuss the relevance of this framework for the representation of opinions, since the value of the diffused parameter can take only two values: 0 or 1 . In work that are maybe closer to social science, this binary representation is often used to study the adoption of a product through imitation of neighbours, like in (DJBJ07). What defines an agent is his state (has adopted, doesn't want to adopt or has not heard of the product yet) and the shape of its utility function, which reveals its tendency to be influenced by others or follow its own desire. The more adopters the agent knows when it decides to adopt, the higher its chance to adopt as well. In this literature, it is possible to relate an artificial population to some observed adoption dynamics, and to assess the diversity of the situation depending on the type of products, for example (DJBJ07). It can be applied to research in marketing in order to suggest guidelines for promotional activities. Some people couple this to market and production dynamics to make the predictions more accurate (MS10).

Another type of simulation, also very popular and refered to as the "Deffuant model", represents an opinion as a real number value, and defines the social influence in an interacting pair using the notion of bounded confidence. Agents are not only defined by the value of their opinion $(o)$, but also by a level of uncertainty $(u)$, where a segment of opinion $([o-u ; o+u])$ contains all the opinions that can influence the agent. An agent with high uncertainty (resp. low) has a low confidence (resp. high) since it can be easily (resp. rarely) influenced. This influence algorithm is such that the influence is not symmetric when the agents do not have the same uncertainty. Uncertainty itself evolves when opinion changes. The features of opinion diffusion that are created by this algorithme are now quite well-known and have been studied largely (DAWF02). In particular, results are largely dependent on the distribution of uncertainty. The fact that in some cases agents cannot be influenced is refered to as bounded confidence (HK02) in this type of representation of an opinion. Changing the definition of bounded confidence can also have an influence on the macro results (HK02).

Eventually, another representation of influence is found in the seminal work of Axelrod (Axe97). In his paper, what we call belief is "culture" 1 and is represented by a bit string of $n$ features, each of which can take different traits (each feature can

\footnotetext{
${ }^{1}$ The choice of terms is always debated in models of influence. Axelrod is very clear on his terminology choice: "Unfortunately, no good term describes the range of things about which people can influence each other. Although beliefs, attitudes, and behavior cover a wide range indeed, there are still more things over which interpersonal influence extends, such as language, art, technical standards, and social norms. The most generic term for the things over which people influence each other is culture. Therefore, the term culture will be used to indicate the set of individual attributes that are subject to social influence." We can conclude that there is no good way or bad way to name these elements that circulate among agents, and this is why we simply adhere to the usual view in ABM influence models.
} 
take the same number of traits). An agent can only influence one of its neighbors; influence occurs when both agent agree on the traits of some features: if they have $60 \%$ of common beliefs, the (more confident) agent has a probability of $60 \%$ of influencing the other agent. One can recognize the same intuition as with the previous notion of bounded confidence, where proximity of beliefs increases the probability of influence, but there is no heterogeneity among agents here. The representation of beliefs in our system is a simple version of the culture of Axelrod with 10 features and two traits fror each feature, and we use the same calculus of a distance between two opinions (Hamming distance). The influence model being very different, and the dimension he studies being absent in our model, we cannot directly compare our results to his. Multidimensional beliefs also appears in (HDJ07) which is an extension of the previously cited "Deffuant model".

Our model can be positioned within this literature. We build a simple model of learning through influence, where the representation of beliefs is close to the one of Axelrod (Axe97). The transmission of beliefs, however, was influenced by the model of (DAWF02) since we consider that the agents have heterogenous ability to influence and be influenced. What has not, to our knowledge, been present previously in research on social influence, is the fact that there exists a "correct" belief, a fixed target that agents could potentially learn about, and that a belief that is extremely "wrong" is more difficult to transmit than a "correct" belief. Our aim is not so much to see if we can achieve convergence of beliefs, but rather to explore whether the convergence of beliefs can also be regarded as collective learning, which enables a better coordination in time. We now describe our model before explaining its main dynamics.

\subsection{Confidence and overconfidence}

As said previously, the notion of bounded-confidence is very present in influence models and can be defined simply: how willing one is to be influenced by another agent. The proximity of beliefs is the key dimension in all previously cited papers: the closer the beliefs, ther easier it is to communicate and influence eachother. Here we describe other uses of the notion of confidence which are related to our central question of learning.

The notion of confidence is found in psychology, and more recently in economics where it is studied greatly to explain anomalies in expected behaviors, in particular when it comes to investment prediction and choices ((Hil03)). The main source of overconfidence is miscalibration, which is the tendency to overestimate the precision of one's information - people tend to state (and act as if) their belief was more certain than it actually is. or example an individual will pretend that he is right with $70 \%$ chance, but reveal himself to be wrong in much more than $30 \%$ of tested cases. The notion of bounded-confidence as defined in the influence literature, and the way we precisely define it in our model, are in line with this notion of overestimation of the quality of your belief. 
It has been widely demonstrated that the tendency to be overconfident is very present in the population, but also heterogenous ((FSL77)). Interestingly it is not linked at all to objective knowledge, being completely decorrelated from IQ for example ((BHMP05), (SW98)). The reason for being overconfident are usually linked to experience, both in the short term and in the long term. For example, it is shown that men are more overconfident than women ((BO01)(SK04)) or that some types of professional training can induce more overconfidence than average ((SK04)). In that sense, it is possible to test overconfidence of individuals with special measures and show that it is stable in time and can be applied to many different tasks, although it varies depending on the domain of the problem and the way the individual has to express its belief ((KSGVB99)). In the short term, the performance regarding a given task also has a huge impact: when being repeatedly successful, an individual will become overconfident, and turn out to be less sensitive to the perception of their following success or errors ((HM06)). Once overconfidence is installed, individuals do not care about the actual result of their choice and do not reevaluate their confidence. This usually leads to an increase of bad choices for the ones that were initially successfull ((HM06)).

Indeed some studies show the impact of overconfidence on success, and it is usually shown to be negative ((CL99)). As ((BHMP05)) explains (we do not cite his references): "Both psychologists and economists have argued that positive ilusions may lead individuals to attaint better outcomes, or example through motivating them to work harder and persiste when the going gets tough. However our experimental results suggest that realism can produce more positive outcomes in market situations in which agents compete and where perspicacity and accuracy in judgment may count for more than motivation and persistence. This is in line with psychological studies which show that realism facilitates performance when acuracy judgment is important for selecting successful effort investment strategies. (..) In sum, the markets studied seem to punish - not reward - miscalibration and positive illusions."

The question we address with our simulation study is in line with this studies. We have a slightly more collective approach since we are not interested in individual performance, but a global observation is used here to decide of the impact of overconfidence on performance - seen as learning of the group about a target reality. In our model, the notion of overconfidence is indeed very close to the one described in psychological literature. How difficult it is for an agent to be influenced may be related to its self-evaluation of the quality of its belief. In our model, it is also heterogenous and totally decorrelated from the actual quality of the belief; an objective reality has a role to play in that success is reduced with bad belief, and overconfidence is not good for adapting to this reality and succeeding in actions. 


\section{Model, simulations and observed indicators}

\section{$2.1 \quad$ Model}

In this model, agents share an environment with some characteristics that they cannot perceive directly but about which they have a belief. They interact by engaging in common actions and learn thanks to these actions. The characteristics of agents and the dynamics of the model is described here, and we give the values parameters can take.

Agents are situated in an environment that is defined by a bit string, each bit taking 0 or 1 as values. It is randomly initiated (each bit takes value 0 or 1 with a 0.5 probability) and stays unchanged throughout the simulation. In the basic version, the length of the bit string is 10. (In more general tests we lead and discuss here, the environment can have other sizes and the bits can take more values but these tests are used to show robustness and limits of the result and we always refer to the "basic" version).

Agents are characterised by their belief about the environment ("belief") and their self-confidence ("confidence"). The belief has exactly the same shape as the environment bit string and is initialised randomly and independently for each agent. Belief evolves through influence and learning. Confidence is fixed for the whole simulation and takes value 1 to 9 .

We use Hamming distance to define the difference between two beliefs: the number of bits that are different among the two compared strings. The distance can take any integer between 0 and 10 as value. We define in the same way the error of agents, as the difference between their belief string and the environment string. In the appendix 6.1 we give some examples of the computation of distances and consequences on influence.

The simulation is made of successive time-steps. At every step, all agents meet by pair, drawn randomly following a uniform law. Each interaction can lead to the transmission of a belief, which depends on four tests:

1. agents determine who will lead the action: there is one leader and one follower - this is based on relative confidence. The agent with the highest confidence is chosen to be the leader. If both agents have the same confidence, both can be chosen with probability 0.5 .

2. agents see if their believes have enough similarity to be able to perform the action together - if the difference of belief (Hamming distance) is lower than the confidence of the follower, he will accept to act with the leader; if it is strictly lower he will not.

3. agents act and discover if the action is successful - this part is not expressed directly as an action in the model but as a probability of success. The success of the action is drawn with a probability that is equal to the error of the leader: the lower the error of the leader, the higher the chance of success.

4. if the action is successful, the leader influences the follower, otherwise, they part 
without influence taking place - when the follower is influenced, he copies a number of bits of the leader's belief to replace his own. The number of bits that are copied is common to all agents and fixed: the "quantity of learning" or $\mathrm{qoL}^{2}$. If the action fails, they split without exchanging any information.

As can be seen, agents have memory neither of their interactions, nor of their preeceding beliefs.

\subsection{Simulations}

We present two types of simulations in this paper, those with a population which is uniform in terms of values of confidence, so that to show the impact of this level of self-confidence on learning. Then we treat simulations with heterogenous population, where agents can have two different confidence levels. We limit the simulation to the description of two populations, due to results of a former work (cite Rouchier, 2007). It has been shown that distribution of values of confidence is what matters in the system, not average value. More precisely, highest and lowest values of confidence are the ones that have the main impact on the dynamics. These simulations are thus not described with "the list of confidence" of agents but just by the size of two groups and the value of the high confidence and the low confidence defining each group. Homogenous populations are of course a special case of this description, with both values of confidence being equal.

A simulation is defined by its initial setting:

- the number of agents (here fixed to 100 for uniform simulations);

- a randomly drawn bit string to describe the environment;

- randomly drawn bit strings, beliefs of all agents;

- the size of the group with high confidence;

- the high value of confidence;

- the low value of confidence;

- the quantity of learning (qoL);

- the way the success is associated to belief (in the above description, the basic model, the probability of failure is linear with error of the leader);

- the size of the bit string that represents the environment (basic model is 10);

- the number of different bits that can be used to represent the environment (basic model is 2).

The indicator we use is the quality of learning of the whole population, which is the average number of correct bits for all agent' believes. Sometimes we also observe the speed of learning which is the speed of reaching the point where agents all agree on their belief.

\footnotetext{
${ }^{2}$ The difference between "quantity of learning", the parameter, and quality of learning, as an indicator, has to be stressed. The quality of belief is the global indicators that gives the average mistake of agents
} 
A simulation is the succession of 1000 time-steps. We are interested in some statistical regularities of the learning process that occur in the medium run. Sometimes, the simulation stops in situations where the believes of agents are not stable, especially when they do not succeed in learning so well. In particular, this will be true when the size of the representation of reality is increased (as seen in the next section) or when agents are all very confident. Logically, the system should reach one of the two possible absorbing states, where agents' beliefs do not evolve anymore. This happens either when agents have identical beliefs or when they cannot undertake common actions anymore (being to distinct in beliefs). Depending on initial conditions and realisations of random variables, learning will stop in one of these states. However, the length of the simulation can be extremely long. In this case, running the simulation for 2000 or 5000 time steps does not improve learning as it is defined: agents' beliefs can improve and then get worse, and get better again, without converging necessarily during this time. In social systems, the opportunities of learning are rather scarce and since we wish the model to be intuitively realistic, we consider that the time of convergence should not be too long and that it is relevant to limit the number of interactions.

In the first set of simulations, the ones with population with uniform confidence, we lead simulations in different direction. First we show the influence of the value of confidence on the learning, which is straighforward in this system. We evaluate the influence of two parameters that are rather critical in this type of representation, as has been noted by Axelrod (Axe97): the size of the representation of the belief and the number of different bits that can be use to build the representation. We also show that two other parameters, one central in our learning dynamics and one in our influence dynamics can be of importance: the probability of success as a function of the error of the leader and quantity of learning. After discussing these results we use the so-called basic model to show our central point with heterogenous populations.

\subsection{General observations}

Each simulation was run about 100 times; results are qualitatively stable with some possible quantitative variations and this is why we average over the simulation runs. Before giving simulation results, we can note a few dynamical characteristics of the model that can be deduced without running the simulations.

- An agent can revise its belief only if it is a follower, which happens only when it meets some other agent with a confidence which is at least higher than its own. Hence: the higher the confidence of an agent, the less it will learn. When two groups of agents have different confidence, the agents with high confidence can only learn from agents of the same group. If this group is small, they cannot learn much.

- In the interaction process, introducing a network that differs from the complete graph would restrain communication to pairs connected in the network. In our 
simulation, an agent can only interact with an agent which has a belief that is close to its own. This limitation induces a network that connects only agents with close beliefs. By introducing heterogeneous confidence we create a non symmetrical "influence network". However this network is never fixed since beliefs evolve all the time. For example, it is clear that when time goes on, agents learn from each other, hence beliefs will converge a bit, then and more more actions will take place, implying more convergence of beliefs. The system displays a dynamic feedback on the speed of convergence through the size of the connected interaction network.

\section{Dynamics of the model with uniform population}

These simulations are special cases where all agents have the same chance of being follower or leader at each time-step, and where the quality of the belief impacts on the action every two time-steps on average. In this case, the number of actions depends directly on the confidence of the population: the higher the confidence, the less agents collectively learn about the environment. After this main result, we test its robustness relative to several parameters. We first give the influence of the qoL (number of exchanged bits) and choose a value that will be stable for the following simulations. Then, we study the way success is related to beliefs in the environment. Eventually, we study the influence of the size of the belief, the number of possible values for each bit (complexification of the reality) and show that the result holds. From this we stabilise our basic setting: the bit string is of length 10 with values being 0 or 1 , qoL is 5 , an the failure of agents is linear with the error of the leader.

\subsection{Influence of confidence}

We give here results based on the previously defined basic design and vary the value of confidence from 1 to 9 . Several patterns can be observed:

1. learning is complete, all agents have a belief that corresponds precisely to the environment bit string after some time;

2. agents learn about the environment, without reaching complete knowledge but with convergence;

3. agents learn a bit about the environment, without converging to a single belief.

4. no learning takes place.

When learning occurs, its dynamics is such that there is a regular increase in the number of correct bits of agents, until it reaches it final value. The number of correct bits reaches 10 in almost all simulations when confidence is lower than 5 . When confidence is 6 or 7 , there is no complete learning but agents' belief converge and get closer to the reality. Eventually, when the confidence value is 8 or 9 , there is little or no learning at all: the number of correct bits stays almost unchanged (see Figure 
1 ), which means similar to the initial random value of 5 . Hence either learning starts or it does not. This is explained quite straighforwardly with the number of actions that can be undertaken, due to the rules of interactions. The number of actions when confidence is low, less than 5, is around 40 at start. At the beginning agents know nothing, so they have random knowledge, which gives a probability of 0.5 of success, and hence the average number of success is 20 . The influence process can take place, which improves knowledge quite quickly and has an impact on the number of possible actions since agents agree more on the characteristics of reality. This is a virtuous circle where convergence of belief increases the ability to act, hence to learn and converge in belief. The same learning process, based on positive feedback, can take place for confidence 6 or 7 , and very occasionally with confidence 8 . In these cases it starts a bit later and accelerate after some time (see Figure 2).

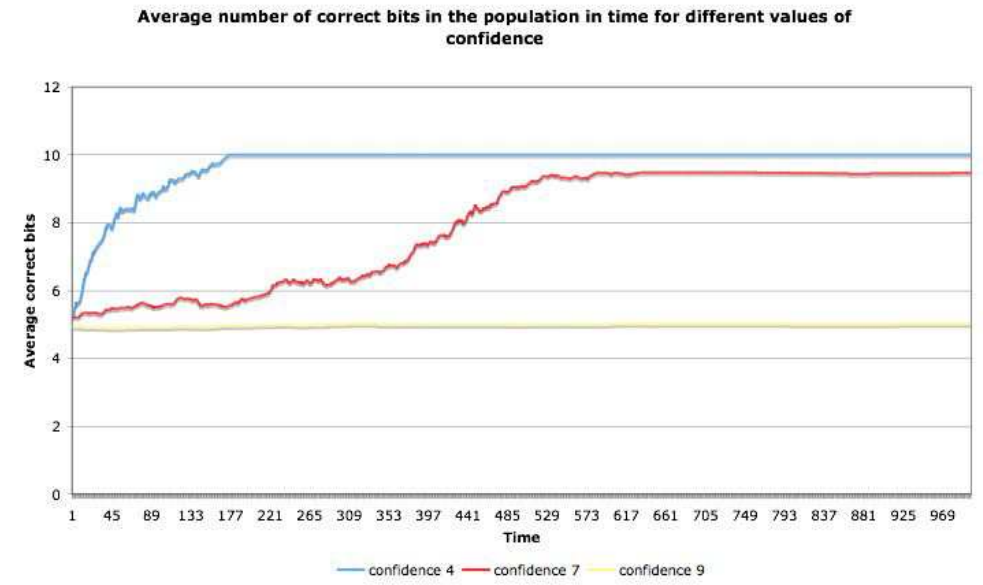

Figure 1: This figure shows results from three typical simulations. It is the evolution over 1000 steps of the number of average correct bits in a population of 100 agents with uniform confidence. Variation of confidence give different dynamics of learning and in particular, when confidence is at 9 , there is no sign of learning in the group.

When uniform confidence increases the time to attain good learning or complete knowledge increases as well. The number of simulations with no good learning also increases: when the confidence is above 7 , it is often the case that the agents do not learn 9 bits.

\subsection{Varying the learning and influence dynamics}

\subsubsection{Quantity of learning}

Quantity of Learning (qoL) has no qualitative impact on our results, apart from two values: when it is equal to 1 and 10. Our first assumption was that changing qoL 


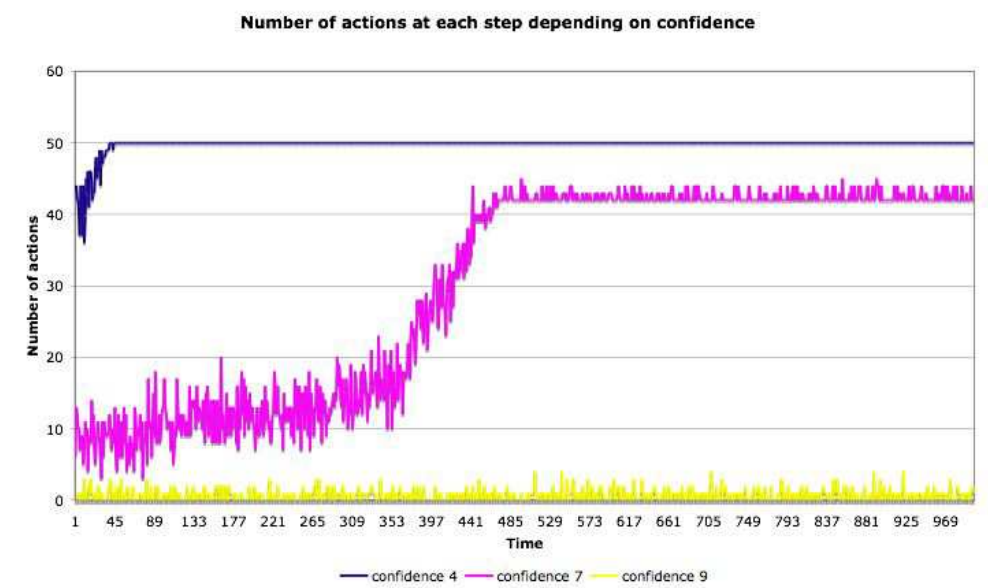

Figure 2: This figure shows the number of actions at each time-step over 1000 steps in a population of 100 homogeneous agents. When confidence is 7 , one can note that takes time to have agents beliefs converge enough to increase significantly the number of actions. After this minimal converge on belief through learning, the possibility of actions increases stiffly.

Table 1: Different values depending on confidence for 100 simulations with basic model: cases without complete learning, cases when final knowledge is less that 8 , time to complete learning and average value when complete learning is not attained.

\begin{tabular}{|l|l|l|l|l|l|l|l|l|l|}
\hline Confidence & $\mathbf{1}$ & $\mathbf{2}$ & $\mathbf{3}$ & $\mathbf{4}$ & $\mathbf{5}$ & $\mathbf{6}$ & $\mathbf{7}$ & $\mathbf{8}$ & $\mathbf{9}$ \\
\hline $\begin{array}{l}\text { Simulations with- } \\
\text { out complete learn- } \\
\text { ing }\end{array}$ & 1 & 0 & 3 & 2 & 17 & 70 & 100 & 100 & 100 \\
\hline $\begin{array}{l}\text { Simulations with no } \\
\text { learning }\end{array}$ & 0 & 0 & 0 & 0 & 1 & 0 & 2 & 97 & 100 \\
\hline $\begin{array}{l}\text { Av. time to com- } \\
\text { plete learning }\end{array}$ & 247 & 232 & 234 & 250 & 263 & 347 & - & - & - \\
\hline $\begin{array}{l}\text { Av. knowledge if no } \\
\text { complete learning }\end{array}$ & 9 & - & 9 & 9 & 9.8 & 9.84 & 9.36 & 6.27 & 5.05 \\
\hline
\end{tabular}


would vary convergence time and we hence ran simulations for 2000 and 5000 timesteps for this test, with no difference in result. In situations where all agents learn easily (100 agents of confidence 1), all values of qoL apart from 1 and 10 make agents learn all 10 bits. When qoL is 1 the average learning over 30 simulations is 9.8 with an MSD of 0.2 and qoL of 10 gives a learning of 8.4 with an MSD of 0.7. Clearly qoL $=1$ gives a results which is slightly different while qualitatively equivalent to other values, whereas $\mathrm{qoL}=10$ changes the results. We have also tested this result for various values of confidence, excluding qoL of 10 , and observing average values and MSD (presented in Table (2)), we conclude that the value of qoL has a non significant impact. However, the value of 1 , which is the one chosen by (MDNW04) gives slightly less go results in our case, and we decided not to use it. Since we were going to have very various type of simulations, with various length of bit strings, we decided to fix qoL that is half the number of bits of the reality string for the following results presented in this paper. One has to remember that agents copy randomly chosen bits that can be identical to the ones they had or different: hence they change at most qoL bits when they get influenced and the closer the beliefs, the smaller the number of bits that are actually changing.

Table 2: Average values of learning and MSD for each value of confidence where qoL changes from 1 to 9 .

\begin{tabular}{|l|l|l|l|l|l|l|l|l|l|}
\hline Confidence & $\mathbf{1}$ & $\mathbf{2}$ & $\mathbf{3}$ & $\mathbf{4}$ & $\mathbf{5}$ & $\mathbf{6}$ & $\mathbf{7}$ & $\mathbf{8}$ & $\mathbf{9}$ \\
\hline $\begin{array}{l}\text { Average of correct } \\
\text { bits }\end{array}$ & 9.95 & 9.94 & 9.95 & 9.97 & 9.92 & 9.67 & 8.64 & 5.74 & 5.04 \\
\hline $\begin{array}{l}\text { MSD comparing } \\
\text { values of qoL }\end{array}$ & 0.06 & 0.07 & 0.07 & 0.04 & 0.1 & 0.35 & 0.99 & 0.32 & 0.04 \\
\hline
\end{tabular}

\subsubsection{Reaction of the environment: changing success of action}

The learning and influence dynamics clearly relies on the probability to achieve a successful action when belief is given. We hence tried to vary this to see if the main result (influence of confidence) would stay true. In all previous examples, the curve was a line, $10 \%$ or good knowledge would give $10 \%$ of success. Now, we test convex and concave curves which have different flatness at extreme ends, as shown in Table 4 and in Figure 8 in appendix 6.2. This should have an impact on transmission of belief.

We again establish two results based on the simulations with the different curves. First, the reaction of the environment does have a huge impact at global level, and it is the one that could be expected. When the reaction of the environment relies on a concave curve of probability, learning do not take place as well as with the linear curve and can even be blocked (Figure 3). Interestingly, these "concave" settings, where a 
Table 3: Probability of success (in percent) for action as a function of the number of bits that the agent knows. We build five different reaction of the environment. The concave ones are such that a bad knowledge can give better success than linear basic model; the concave ones are such that it needs good knowledge to increase probabilty of success.

\begin{tabular}{|l|l|l|l|l|l|l|l|l|l|l|l|}
\hline $\begin{array}{l}\text { Number } \\
\text { of known } \\
\text { bits }\end{array}$ & $\mathbf{0}$ & $\mathbf{1}$ & $\mathbf{2}$ & $\mathbf{3}$ & $\mathbf{4}$ & $\mathbf{5}$ & $\mathbf{6}$ & $\mathbf{7}$ & $\mathbf{8}$ & $\mathbf{9}$ & $\mathbf{1 0}$ \\
\hline Linear & 0 & 10 & 20 & 30 & 40 & 50 & 60 & 70 & 80 & 90 & 100 \\
\hline Convex 1 & 0 & 5 & 10 & 16 & 23 & 30 & 39 & 49 & 60 & 72 & 100 \\
\hline Convex 2 & 0 & 1 & 2 & 3 & 5 & 8 & 12 & 20 & 30 & 55 & 100 \\
\hline Concave 1 & 0 & 20 & 35 & 50 & 60 & 68 & 75 & 84 & 90 & 95 & 100 \\
\hline Concave 2 & 0 & 40 & 60 & 70 & 80 & 85 & 91 & 95 & 97 & 98 & 100 \\
\hline
\end{tabular}

bad knowledge has a chance of being transmitted, even has a dynamics which is different from all previously observed dynamics: it is possible to see kwoledge improve and then regress. We did not try to understand the reason of this result for this paper. Neither did we try to find a limit case of concavity, where the concave curve could still imply good learning in the setting we chose for the shown simulations. What interests us here, once more is to show that the "basic" setting, here with linear reaction, although not universal, is not the peculiar that it would not be extendable. Clearly, our learning mechanism still works in environments where error in representation makes it harder to succeed and transmit a knowledge (convex reactions).

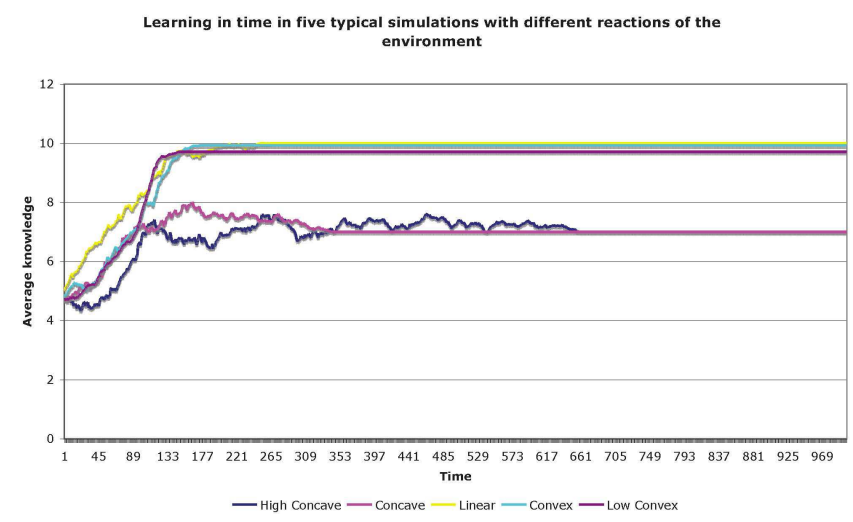

Figure 3: This figure shows the evolution of the number of correct bits on average in a population of 100 agents of confidence 5, with qoL 5, for our five reactions of the environment. Concave reactions clearly display dynamics that are different from the others. It shows that our learning model is not appropriate in environments where it is possible to act successfully with bad knowledge. 
The second result is the robustness of the characteristics of the model we are interested in: the dependence of learning to confidence is again witnessed, as well as the fact that learning is equivalent for all values which are less than 5 and that confidence above 8 implies very bad learning.

Table 4: Average knowledge of agents at the end for 50 simulations, with different reactions of the environment and confidence. Increasing confidence reduces learning.

\begin{tabular}{|l|l|l|l|l|l|l|l|l|l|}
\hline & $\mathbf{1}$ & $\mathbf{2}$ & $\mathbf{3}$ & $\mathbf{4}$ & $\mathbf{5}$ & $\mathbf{6}$ & $\mathbf{7}$ & $\mathbf{8}$ & $\mathbf{9}$ \\
\hline High Concave & 8.8 & 8.51 & 8.77 & 8.2 & 8.02 & 8.27 & 8.12 & 5.5 & 4.96 \\
\hline Concave & 9 & 9 & 8.4 & 8.6 & 8.8 & 8.67 & 8.38 & 5.86 & 5 \\
\hline Linear & 10 & 10 & 10 & 9.99 & 9.98 & 9.89 & 9.31 & 6.49 & 4.95 \\
\hline Convex & 10 & 10 & 10 & 10 & 9.97 & 9.72 & 8.88 & 5.92 & 5.01 \\
\hline Low Convex & 10 & 10 & 9.98 & 9.94 & 9.68 & 8.87 & 7.64 & 5.48 & 5.12 \\
\hline
\end{tabular}

\subsection{Varying the representation of the environment}

We vary two parameters in the representation of the environment, following Axelrod (Axe97) in his seminal paper. We modify the number of bits of the bit string and increase the number of possible values that each bit can take. Since our model is rather simple at the start (1024 different possible reality strings), we mainly test the influence of an increase of complexity.

\subsubsection{Length of bit string}

When we change the length of the bit string, where bits can take value 0 or 1 , two main results appear. First, the influence of the confidence of the population is still the same. When confidence increases, population learning gets worse, and above a certain threshold, learning do not take place at all. This is shown by Figure (4). The second result is that the complete learning cannot be achieved anymore when the size of the bit string increases too much. Even in the best conditions, with confidence of 1 and with an increased simulation time (5000), agents do not learn completely, although they can converge to the same belief. This can be seen in Figure (5) where the actual learning is compared to complete learning.

\subsubsection{Number of possible bits}

We test the influence of the number of possible bits on the learning of agents. In our basic model, there are just 2 values, 0 and 1, which allows 100 different possible environment to exist. We test the learning of the 100 agents with different values of confidence, with qoL of 5 . Two results are observed. First, the value of confidence once more has an impact on learning in this system, as can be seen on Figure (6). However, 


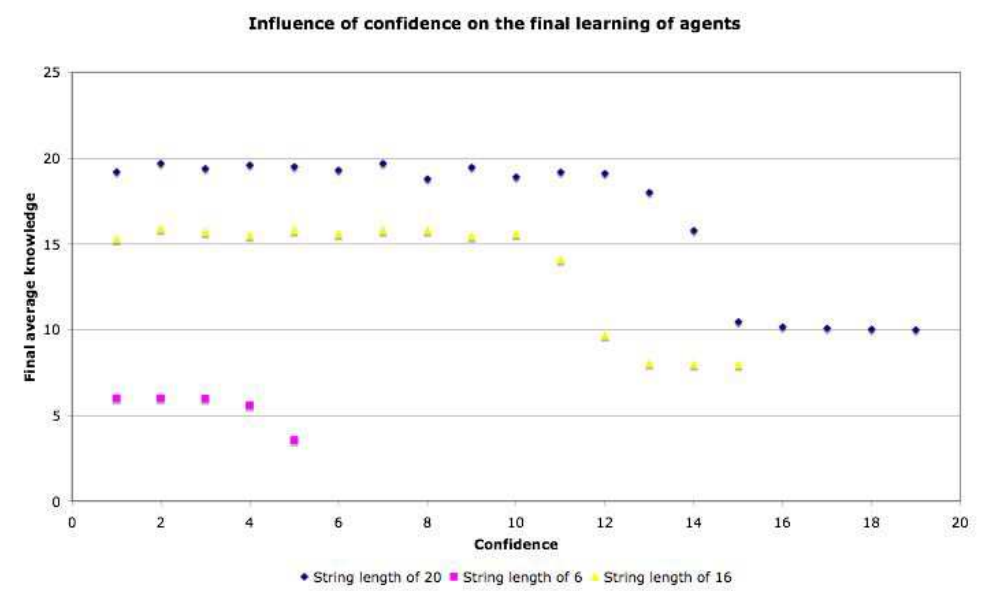

Figure 4: This figure shows the evolution of the number of correct bits on average in a population of 100 agents with all values of confidence, for different values of string size. Confidence is tested for values from 1 to (string size - 1). The qoL is half of the string size. For all the situations shown here the final learning of agents is directly linked to the confidence.

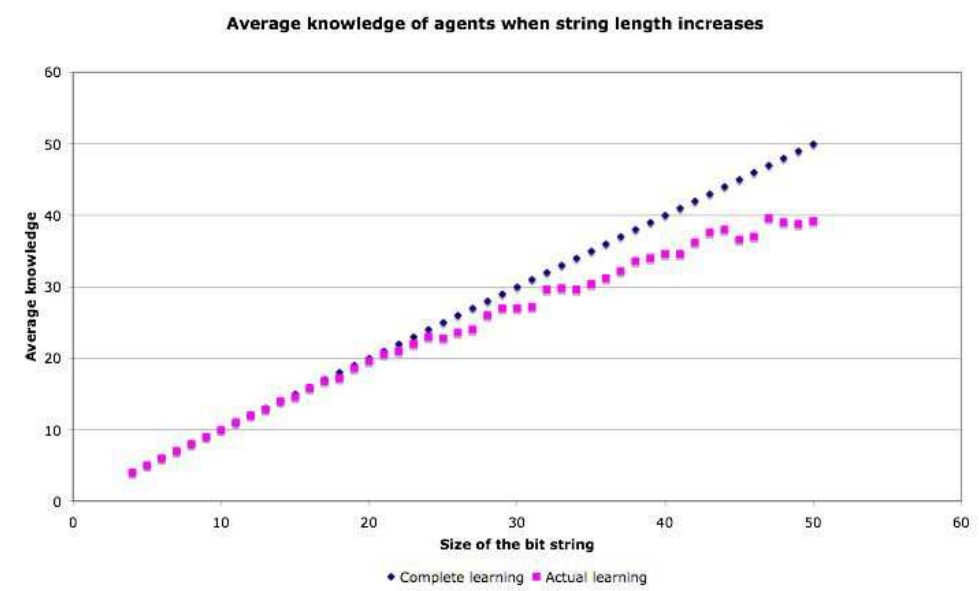

Figure 5: This figure shows the average learning after 1000 up to 5000 time steps (depending on the length of the bit string) of learning for agents of confidence 1, with qoL of half the size of the bit string. It has to be noted that there is no difference when siulations runs for 2000 or 5000 time-steps for high lengths of the string, meaning that the society stabilises in a situation where learning is not so good. 
as the number of possible values for each bits increases from 2 to 10, confidence need to be smaller to enable agents to learn. In cases the system is really complex (10 bits taking 10 different values, so 10000000000 different environment are possible) only agents with confidence of 1 can learn the environment almost perfectly. A table gives in appendix (ref à venir) gives all values with the number of values varying from 1 to 10 .

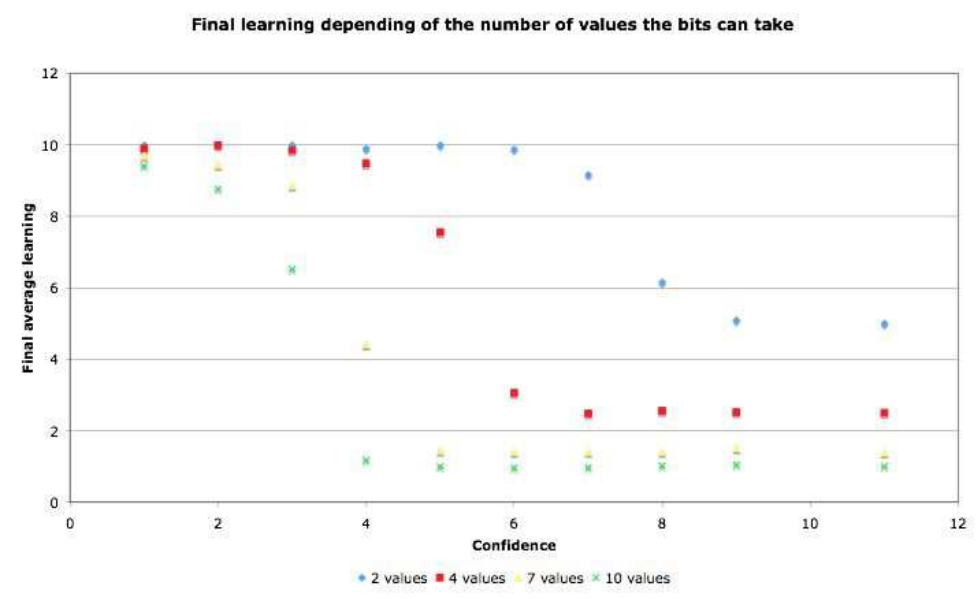

Figure 6: This figure shows the average learning after 1000 for agents of confidence 1 to 9 where the number of possible values for each bit of the bit string changes. When confidence increases, learning does not happen at all, as can be seen by comparing the number of correct bits to the one that is corresponds to the initial random attribution of belief (varying with the number of possible values - on the far right of the figure).

\subsection{A short discussion}

Before turning to simulation with two populations of different confidence, it can be useful to draw a few conclusions on aspects that do interest us in all previous results. We are interested in three aspects.

- Our basic setting, which is rather simple, represents a good model for our aim. It does dicriminate among simulations based on the criteria we are interested. It is not too sensitive to the main criteria which we cannot justify and which would be, in real life, certainly impossible to measure in any way: the quantity of learning.

- This basic setting is very specific and simple. However, increase in complexity in two ways do not destroy the model's dynamics; change of the environment reaction completely changes the learning dynamics if error is less "punished" than in the basic model. We can then trust our model to display some generality. 
This is interesting in the perspective of potentially trying to consider our results as conclusive for general environmental issues, as we said in introduction. Of course, fitting the model to otside reality could be rather complex, but at least the possibility is not rejected at this level.

- Up until now our model gives global and dynamical results that are in line with the individual definition of confidence and overconfidence as defined by psychologists. It seems reasonable to use it to explore the impact of one of their observation: the heterogenity of this confidence in population. Again, we are mostly interested in collective view, rather than individual, and for the moment our agent-based model seems to fit our aim.

\section{Dynamics with two confidence levels}

We now turn to simulations that gather two populations of different confidence. In the basic setting we use from now on, two values of confidence have been identified as thresholds. With confidence under 5 all simulations are similar; with confidence above 8 there is almost no learning in the population. We can hence assume that agents of confidence 8 and 9 can be seen as overconfident. We will then proceed by mixing agents of different confidence. We will see how very few overconfident agents reduces collective learning (mixing agents of confidence 4 and 8 ). We will also study the possibility that heterogeneity is the reason of this reduction of learning, not just overconfidence (by mixing agents of confidence 1 and 4). Once this hypothesis has been tested, we will also observe that agents who usually learn well when they are in an homogenous population can be led to underperform when they meet agents with low confidence (mixing agents of confidence 3 and 7 ). Eventually, we will check that these results are stable if we change the interaction rule slightly: we will add some random element to the choice of the leader by allowing low confidence agents to sometimes influence high confidence agents. This will assure us, again, that the model can have a bit of genericity.

\subsection{Presence of overconfident agents}

When the group of high confidence agents represents more than $15 \%$ of the population, the situation is similar to one where all agents have a high confidence.

Hence, the size of the network with whom the agents can interact is central in this system. The higher the confidence of an agent the smaller the network that can influence him (two constraints have to be met: at least same confidence and more and more similarity as confidence grows). The result we observe in the system is still true for values of confidence that are higher ( 8 et 9 ) and the higher the confidence, the more impact on learning for agents with low confidence. 


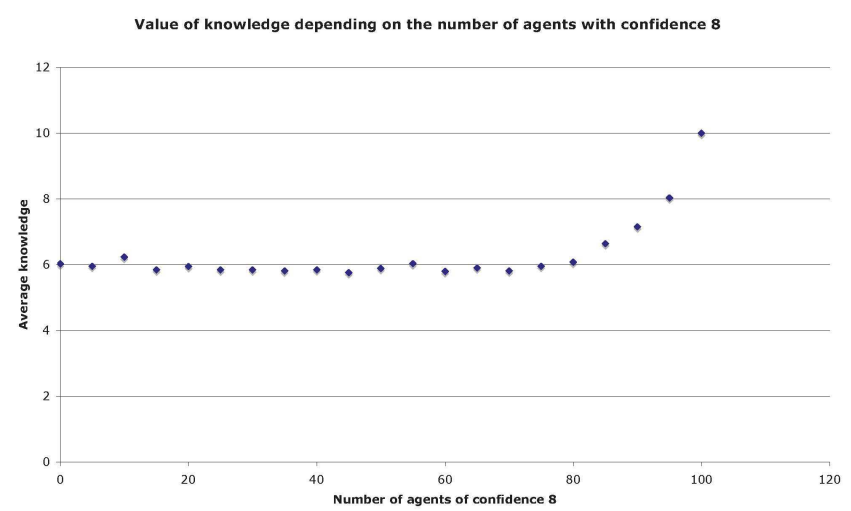

Figure 7: Average number of correct bits after 5000 time-steps in a population of 100 agents, for all possible values of the number of agents with confidence of 7 . All other agents have confidence of 2 . The results are given for the whole society, as well as for both groups of different confidence. A small number of agents with high confidence reduces the quality of learning, up until $20 \%$. Above $20 \%$, the learning gets better as the number of high confidence agents increases..

\subsection{Influence of simple heterogeneity}

As was said before, using two populations in the simulations creates a subgroup of agents who can only be influenced by eachother. Hence we add the effect of overconfidence to reduce learning, but also the fact that agents with higher confidence can only learn from agents with the same confidence as theirs. One can imagine that the reduction of the size of the possible influence also reduces the chance to get correct information about the belief. We should then see if heterogenity itself is not a cause for bad learning. For this, we just mix populations who usually achieve good learning, agents with confidence 1 and 4 . Observation of simulations show two facts. First, when the number of high confidence agents is higher than 20, the only difference is that the learning is longer than in usual simulations, but gets to complete knowledge most of the time. When higher confidence agents are in low number, however, the learning gets bad (Table ). Hence, to have bad learning in our system, there is no need to have overconfident agents.

Facing this issue the best solution to repair the bad learning would be to increase the size of the population - in that case agents of confidence 4 could share more information and get to better knowledge. We test the impact of increasing the size of the population for both mix we presented before, 1-4 and 4-8, with results after 2000 time-steps. 


\subsection{Adding noise in influence}

A second way to suppress the bad collective learning when it is due to group size effect, as shown previouly, would be to keep the same size of population, but allow agents

What these

\section{Conclusion}

In this paper we describe a model of collective learning about an objective target, which is locally based on social influence. The presence of this objective target is the main difference between our model and usual dissemination models, except maybe (WDAN02). We use a notion of overconfidence to describe our agents: these agents can indeed be very hard to influence because they overestimate the quality of knowledge they have about their environment. As a consequence they cannot learn so well as individuals. We wonder here if this individual overconfidence has an influence at the scale of the whole population.

We first study the robustness of our learning model, by varying many parameters of the system. First we can note that, in a homogenous population, the higher the confidence, the worse the learning. This study also shows that the model, although very simple, can produce results that stay true in more complex settings. We then use the same model to study the heterogeneity of confidence and in particular the presence of overconfident agents. These very confident agents do not learn the characteristics of a fixed target very well, but they also disturb the learning of low confidence agents.

In particular for values of 8 or 9 , learning does not take place at all in our setting. When two groups exist, the presence of heterogeneity reduces agents' ability to learn. The probability of meeting another agents by whom to be influenced is the central point: indeed, when the number of high confidence agents increases, they get closer to a uniform situation and can learn among themselves, having less bad influence on low confidence agents. We spotted that the worst proportion of high confident agents is about $20 \%$. Hence, a group that has to learn about a form of reality can be stopped in the learning by a small group of very confident people who will learn less rapidly.

TROUVER BURA ET LA MEMETIC ou Hales An open mind is not an empty mind.

By going through an analytical model whose properties will be explored, we could spot one very important element of the characteristic of the environment. The more the environment discriminates the quality of beliefs - by imposing a signifiant difference in success between agents whose beliefs are more or less accurate, we increase drastically the global quality of learning.

It would be interesting, for further work, to analyze the same learning model in an environment where the actions of agents transform the environment. In that setting, the role of very confident agents might not be as "negative" on learning, since they 
might be the origin of changes that woul lead the environment to ressemble their own belief. The main problem of this new setting would be to decide how actions would change the environment. One could imagine that a succesful action only transforms it, or on this opposite, a failed action only.

Our results are in contradiction with other work, like (WDAN02), where low confidence agents can make high confidence agents learn very slowly. The main reason is of course that in our model, a low confidence agent can on no occasion influence a high confidence agent. Maybe this assumption should be relaxed a bit, for example by adding a very small probability that low confidence agents could influence high confidence ones. It is not sure that this would add much to our model, considering our interest in this dynamics. Alternatively, we could imagine that agents' confidence is transformed through an endogenous evolution. Indeed, agents having very low success (resp. high) in their actions could decide to lower their confidence on some occasions (resp. increase). This would be in line observations on investment behaviours (HM06), which show that individual who know some success can become extremely overconfident, and stay so even if their success fades away. One could also imagine that individuals would become more confident if they often interaction with agents who aggree with them. These two modifications could indeed be more relevant for adapting our model to real life situation, and should certainly be addressed in our future work.

Before doing this, we wish to show with the analytical model that our model is robust to the size of the population, and see in which settings the result is true. We already show that not only agents with good knowledge are important, but also agents with average knowledge, since they are the only ones to communicate with agents who know very little. In our simulation model, we could not detect this, since the beliefs are drawn randomly at start and we did not particularly correlate confidence to beliefs.

Opening : changing environment !!!

\section{References}

[Axe97] Axelrod. The dissemination of culture. a model with local convergence and global polarization. Journal of conflict resolution, 41(2):203-226, April 1997.

[BHMP05] B. Biais, D. Hilton, K. Mazurier, and S. Pouget. Judgemental overconfidence, self-monitoring, and trading performance in an experimental financial market. Review of Economic Studies, 72(2):287-312, 2005.

[BO01] B. M. Barber and T. Odean. Boys will be boys: Gender, overconfidence, and common stock investment. The Quarterly Journal of Economics, 116(1):261-292, 2001. 
[CL99] C. Camerer and D. Lovallo. Overconfidence and excess entry: and experimental approach. American Economic Review, pages 306-318, 1999.

[CR05] P. Chen and S. Redner. Majority rule dynamics in finite dimensions. Physical review E, 71:036101, 2005.

[DAWF02] Guillaume Deffuant, Frederic Amblard, Gerard Weisbuch, and Thierry Faure. How can extremism prevail? a study based on the relative agreement interaction. JASSS, 5(4), 2002.

[DJBJ07] S.A. Delre, W. Jager, T.H.A. Bijmolt, and M.A. Janssen. Targeting and timing promotional activites: an agent-based model for the takeoff of new products. Journal of business research, 60:826-835, 2007.

[FS06] S. Fortunato and D. Stauffer. Computer simulations of opinion and their reactions to extreme events. In S. Albeverio, V. Jentsch, and H. Kantz, editors, Extreme events in nature and society. Springer, Berlin Heidelberg, 2006.

[FSL77] B. Fischhoff, P. Slovic, and S. Lichtenstein. Knowing wih certainty: the appropriateness of extreme confidence. Journal of experimental psychology: human perception and performance, 3(4):552-564, 1977.

[Ga197] Serge Galam. Rational group decision making: a random field ising model at $\mathrm{t}=0$. Physica A, 238:66-80, 1997.

[HDJ07] S. Huet, G. Deffuant, and W. Jager. Rejection mechanism in 2d bounded confidence provides more conformity. In European Conference on Complex Systems'07 (ECCS'07),, Dresden,, October 2007.

[Hil03] D. Hilton. Psychology and the financial market. In I. Brocas and J.D. Carrillo, editors, The psychology of economic decision, volume 1 - Rationality and well-being, chapter 14, pages 273-297. Oxford University Press, 2003.

[HK02] Rainer Hegselmann and Ulrich Krause. Opinion dynamics and bounded confidence: models, analysis and simulation. JASSS, 5(3):http://jasss.soc.surrey.ac.uk/5/3/2.html, June 2002.

[HM06] G. Hilary and L. Menzly. Does past success lead analysts to become overconfident? Management science, 52(4):489-500, April 2006.

[Kre90] David M. Kreps. A Course in Microeconomic Theory. Princeton University Press, 1990.

[KSGVB99] J. Klayman, J.B. Soll, C. Gonzales-Vallejo, and S. Barlas. Overconfidence: it depends on how, what and whom you ask. Organizational Behavior and Human Decision Processes, pages 216-247, 1999. 
[MDNW04] S. Martin, G. Deffuant, J-P. Nadal, and G. Weisbuch. Comparing the binary opinion vector version of the bc model with different approximating aggregated dynamics. In M2M: Model-to-Model Workshop, Valladolid, Spain, 2004. ESSA Conference.

[Mos79] Serge Moscovici. Social influence and social change. London ; New York, 1979.

[MS10] T. Meyer and F. Stöppler. Does the winner take it all? technology diffusion and platform competition in the smartphone industry. In International Workshop on Agent-based Simulation of Diffusion Processes, Vienna, Austria, April 8-9 2010.

[MV72] Humberto Maturana and Fancisco Varela. Autopoesis and cognition. The realization of the living. D. Reidel Publishing company, Dortrecht, Holland, 1972.

[RS07] J. Rouchier and H. Shiina. Learning and belief dissemination through coaction. In S. Takahashi, D. Sallach, and J. Rouchier, editors, Advancing Social Simulation. The first World Congress, pages 225-236. Springer, Tokyo, 2007.

[SK04] J.B. Soll and J. Klayman. Overconfidence in interval estimates. Journal of Experimental Psychology: Learning, Memory, and Cognition, 30(2):299-314, 2004.

[Sta05] Dietrich Stauffer. Sociophysics simulations ii: opinion dynamics. 2005.

[SW98] K.E. Stanovitch and R.F. West. Individual difference and rational thought. Journal of experimental psychology: general, 127:161-188, 1998.

[SWS00] Katarzyna Sznajd-Weron and Jozef Sznajd. Opinion evolution in closed community. International Journal of Modern Physics C, 11(6):1157$1165,2000$.

[WDAN02] G. Weisbuch, G. Deffuant, F. Amblard, and Jean-Pierre Nadal. Meet, discuss, and segregate! Complexity, 7(3):55-63, 2002.

\section{Appendix}

\subsection{Examples of influence}

Two agents, $\mathrm{A}$ and $\mathrm{B}$ interact in environment $\mathrm{E}$, quantity of learning is $2 . \mathrm{E}=\left(\begin{array}{lll}0 & 0 & 1\end{array}\right.$

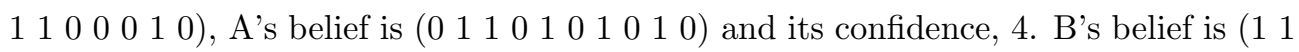
10001000 ) and its confidence is 8 . When they meet, B could lead the action; they have 7 common bids, which is higher than 4 , and hence $\mathrm{A}$ follows $\mathrm{B}$ in the action. $\mathrm{B}$ 
has 4 right bits, which means he has $40 \%$ chance of success. If it succeeds, A copies 2 bits from B to transform its belief (for example it can transform into (1 1110101010 $10)$ ). If $B$ fails, nothing happens.

Two agents, $\mathrm{A}$ and $\mathrm{B}$ interact in environment $\mathrm{E}$, quantity of learning is $2 . \mathrm{E}=(0$ 011100010 ), A's belief is (0 110101010$)$ and its confidence, 8. B's belief is

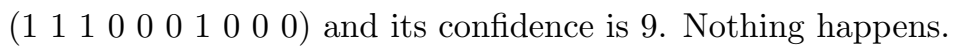

Two agents, $\mathrm{A}$ and $\mathrm{B}$ interact in environment $\mathrm{E}$, quantity of learning is $3 . \mathrm{E}=(0$

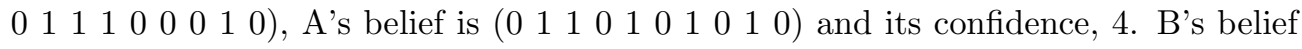

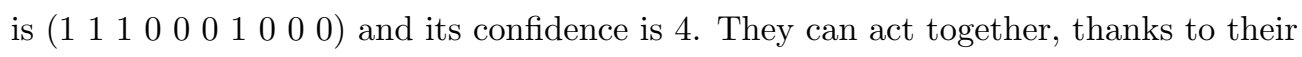
low confidence. We choose with $50 \%$ probability the leader, which is A. it has 7 bits in common with the environment, which means $70 \%$ chance of success. If it succeeds, B copies 3 bits from A to transform its belief (for example it can transform into (0 1

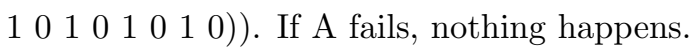

\subsection{Probabilities}

Figure 8 gives a visual representation of the values of the probabilities of the environment for each number of correct bits. The more concave the reaction, the more discrimination it puts between completely wrong and rather wrong beliefs, and slighlty true knowoledge allorws to have some success in action. The more convex the reaction, the more it "punishes" error, even limited.

\subsection{Reaction of the environment}

Table 5 and 6 give average values for 30 simulations of the average correctness in the population over the last 100 time-steps out of 5000. We compare here the learning with linear reaction of the environment and "convex2" reaction, the one which is the flatter at the origine but steeper when approaching complete belief. The comparison shows that in all cases where populations are heterogenous with $80 \%$ of low confidence agents and $20 \%$ of high confidence agents, the population learns better when facing the more discriminatory probabilist rule, and that the simulation display less variation (so there is less influence of initial condition - the only difference in initial condition being the distribution of beliefs). 


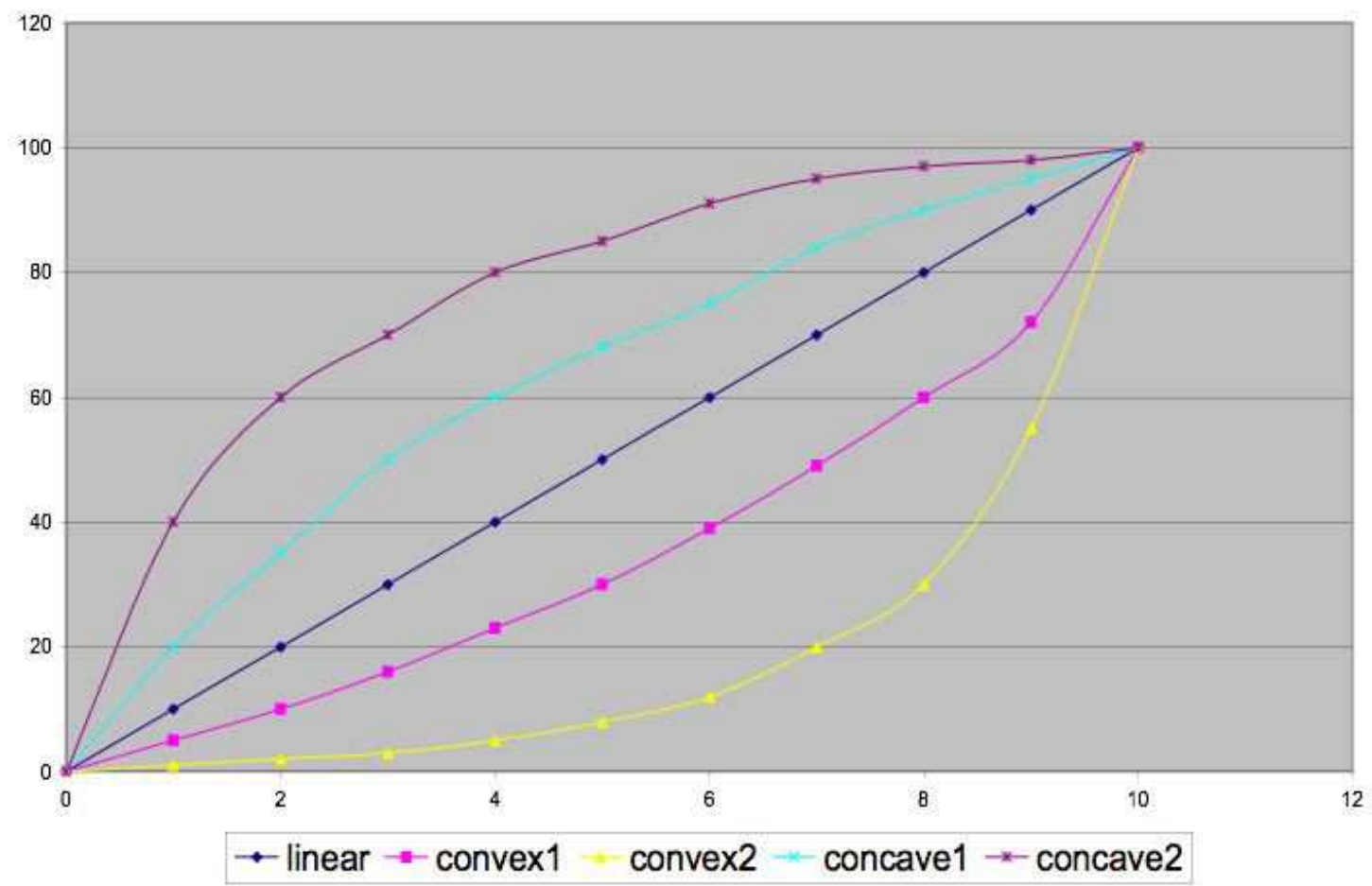

Figure 8: Representation of the probability of success depending on the number of correct bits of the belief. When the curve is convex, there is a high discrimination between knowing very well and well and less otherwise; when the curve is concave, there is a high discrimination between knowling very bad and bad, and less otherwise. 
Table 5: Average knowledge after 5000 time-steps for 200 agents, qol = 2, linear learning (average over 10 simulations). Two populations : 160 with low confidence and 40 with high confidence. Linear reaction.

\begin{tabular}{|l|l|l|l|l|l|l|l|l|l|}
\hline Confidence & $\mathbf{1}$ & $\mathbf{2}$ & $\mathbf{3}$ & $\mathbf{4}$ & $\mathbf{5}$ & $\mathbf{6}$ & $\mathbf{7}$ & $\mathbf{8}$ & $\mathbf{9}$ \\
\hline $\mathbf{1}$ & 10 & - & - & - & - & - & - & - & - \\
& $(0)$ & & & & & & & & \\
\hline $\mathbf{2}$ & 10 & 10 & - & - & - & - & - & - & - \\
& $(0)$ & $(0)$ & & & & & & & \\
\hline $\mathbf{3}$ & 9.7 & $9(0)$ & 10 & - & - & - & - & - & - \\
& $(0.1)$ & & $(0)$ & & & & & & \\
\hline $\mathbf{4}$ & 10 & 10 & $8(0)$ & 10 & - & - & - & - & - \\
& $(0)$ & $(0)$ & & $(0)$ & & & & & \\
\hline $\mathbf{5}$ & $9(0)$ & 10 & $9(0)$ & 10 & 10 & - & - & - & - \\
& & $(0)$ & & $(0)$ & $(0)$ & & & & \\
\hline $\mathbf{6}$ & 10 & 9.8 & 8.9 & 9.9 & 8 & 10 & - & - & - \\
& $(0)$ & $(.2)$ & $(.2)$ & $(.2)$ & $(.2)$ & $(0)$ & & & \\
\hline $\mathbf{7}$ & 8.3 & 8.7 & 7.2 & 9.9 & 7.1 & 8.8 & 9.4 & - & - \\
& $(.3)$ & $(.2)$ & $(.2)$ & $(.2)$ & $(.2)$ & $(.2)$ & $(.1)$ & & \\
\hline $\mathbf{8}$ & 5.4 & 6.9 & 5.6 & 6.7 & 5.6 & 7.6 & 7.9 & 8.5 & - \\
& $(.2)$ & $(.2)$ & $(.2)$ & $(.2)$ & $(.3)$ & $(.3)$ & $(.3)$ & $(.1)$ & \\
\hline $\mathbf{9}$ & 6.4 & 6.2 & 6 & 6.3 & 6.5 & 5.6 & 6.6 & 5.9 & 5.1 \\
& $(.2)$ & $(.3)$ & $(.3)$ & $(.3)$ & $(.3)$ & $(.3)$ & $(.3)$ & $(.3)$ & $(.1)$ \\
\hline
\end{tabular}


Table 6: Average knowledge after 5000 time-steps for 200 agents, qol = 2, linear learning (average over 10 simulations). Two populations : 160 with low confidence and 40 with high confidence. "Convex2" reaction.

\begin{tabular}{|l|l|l|l|l|l|l|l|l|l|}
\hline Confidence & $\mathbf{1}$ & $\mathbf{2}$ & $\mathbf{3}$ & $\mathbf{4}$ & $\mathbf{5}$ & $\mathbf{6}$ & $\mathbf{7}$ & $\mathbf{8}$ & $\mathbf{9}$ \\
\hline $\mathbf{1}$ & 10 & - & - & - & - & - & - & - & - \\
& $(0)$ & & & & & & & & \\
\hline $\mathbf{2}$ & 10 & 10 & - & - & - & - & - & - & - \\
& $(0)$ & $(0)$ & & & & & & & \\
\hline $\mathbf{3}$ & 9.9 & 10 & 10 & - & - & - & - & - & - \\
& $(0)$ & $(0)$ & $(0)$ & & & & & & \\
\hline $\mathbf{4}$ & 10 & 10 & 10 & 9.9 & - & - & - & - & - \\
& $(0)$ & $(0)$ & $(0)$ & $(0)$ & & & & & \\
\hline $\mathbf{5}$ & 10 & 10 & 9.9 & 10 & 9.9 & - & - & - & - \\
& $(0)$ & $(0)$ & $(0)$ & $(0)$ & $(0)$ & & & & \\
\hline $\mathbf{6}$ & 9.9 & 8.8 & 9.6 & 9.8 & 9.2 & 9.8 & - & - & - \\
& $(0)$ & $(.1)$ & $(.1)$ & $(0)$ & $(.1)$ & $(.1)$ & & & \\
\hline $\mathbf{7}$ & 8.6 & 8.9 & 9.7 & 8.7 & 9 & 9.4 & 8.3 & - & - \\
& $(.1)$ & $(.1)$ & $(.1)$ & $(.1)$ & $(.1)$ & $(.1)$ & $(.2)$ & & \\
\hline $\mathbf{8}$ & 8.6 & 8.6 & 8.7 & 8.8 & 8.8 & 8.8 & 8.4 & 6.3 & - \\
& $(.1)$ & $(.1)$ & $(.1)$ & $(.1)$ & $(.1)$ & $(.1)$ & $(.1)$ & $(.2)$ & \\
\hline $\mathbf{9}$ & 8.5 & 8.6 & 8.6 & 8.6 & 8.8 & 8.8 & 8.2 & 7 & 5.1 \\
& $(.1)$ & $(.1)$ & $(.1)$ & $(.1)$ & $(.1)$ & $(.1)$ & $(.2)$ & $(.1)$ & $(.1)$ \\
\hline
\end{tabular}

